\title{
Ethnic and sex differences in the total and differential white cell count and platelet count
}

\author{
Barbara J Bain
}

\begin{abstract}
Aim/background-Previous studies have suggested that healthy subjects of African ancestry have lower total white cell counts, neutrophil counts and platelet counts than Caucasian subjects and that, at least among Caucasians, women have higher neutrophil and platelet counts than men. The primary aim of this study was to confirm and quantify the ethnic differences, confirm the sex difference in Caucasians and determine whether there was a similar sex difference in non-Caucasians. A secondary aim was to establish reference ranges for white cell and platelet counts for the different ethnic and sex groups.
\end{abstract}

Methods-The study population comprised 417 healthy volunteers (201 women and 216 men), of whom 200 were Caucasian, 102 were Afrocaribbean and 115 were African. Full blood counts, including a differential white cell count, were measured using a H.2 automated differential counter. White cell and platelet counts were compared between the three different ethnic groups and between men and women. Reference ranges were determined for each ethnic and sex group.

Results-Africans and Afrocaribbeans had lower total white cell, neutrophil and platelet counts than Caucasians and counts were lower in Africans than in Afrocaribbeans. Women had higher neutrophil and platelet counts than men in all ethnic groups.

Conclusions-Sex and ethnic origin should be taken into consideration when assessing white cell and platelet counts. (F Clin Pathol 1996;49:664-666)

Keywords: normal ranges, ethnic groups, white cell count, platelet count.

Reference ranges for many haematological variables are influenced by ethnic origin and sex. A number of studies have found that total white blood cell (WBC) and, when investigated, neutrophil counts are lower in Africans and those of African ancestry than in Caucasians. Such observations have been made in Zambians, ${ }^{1}$ Ugandans ${ }^{2}$ and Malians, ${ }^{3}$ in South African Bantus ${ }^{4}$ and the Basotho population of Lesotho, ${ }^{5}$ in Africans and Afrocaribbeans resident in Britain, ${ }^{67}$ and in Black Americans. ${ }^{8} \mathrm{~A}$ smaller number of studies have suggested that the platelet count is also lower in Africans
(Zambians, Nigerians and Kenyans) $)^{9-11}$ and in Afrocaribbeans. ${ }^{12}$ In addition, women have $\stackrel{\varnothing}{\varrho}$ been observed to have higher white cell counts, neutrophil counts and platelet counts than $\vec{\circ}$ men. ${ }^{13}{ }^{14}$ In most published studies it was not possible to carry out simultaneous investiga- $\bar{\omega}$ tions of subjects of different ethnic origin and a variety of different methodologies were used, making it difficult to compare results in different ethnic groups. In many studies no allowance was made for the non-Gaussian distribution of total white cell, platelet and differential 을 counts, and differential counts were performed on only 100 cells. The fact that St Mary's Hospital now has many staff of African and Afrocaribbean origin provided the opportunity $\stackrel{5}{\rightarrow}$ to carry out a systematic investigation of the $\vec{\theta}$ total and differential white cell counts and $\mathscr{\mathscr { C }}$ platelet counts in healthy volunteers of various ethnic origins, using an automated counter which also provides a differential count and a measurement of mean platelet volume. At the same time it was possible to investigate $\frac{\mathbb{D}}{\circ}$ whether the sex differences previously ob- $\overrightarrow{\vec{P}}$ served in Caucasians were also present in African and Afrocaribbean subjects.

\section{Methods}

Venous blood samples were obtained from 417 volunteers (201 women and $216 \mathrm{men}$ ), 200 of whom were Caucasian, 115 African and 102 Afrocaribbean. The ages of the subjects ranged from 18 to 55 years. Blood samples were collected over a five year period. The Cauca- $\frac{7}{0}$ sians were predominantly Northern European but a minority were from the Indian subconti- $\tilde{\sigma}$ nent. The Africans were mainly from sub- N Saharan Africa (excluding South Africa), N specifically from Nigeria, Ghana, Ivory Coast, Sierra Leone, Zaire, Angola, Zimbabwe, and 0 Zambia. A minority were from Ethiopia, Somalia and the Sudan, but were identifiable $\stackrel{?}{+}$ and identified themselves as "Black". The vol- Tㅜㅜ unteers considered themselves to be healthy and free of intercurrent infection. Smoking $\stackrel{\odot}{\oplus}$ habits were documented for the first $153 \mathrm{Cau}-$ casian volunteers, comprising 51 male and 51 ㅇ female non-smokers and 26 male and 25 \& female smokers. The smokers smoked a median of 15 (range 5-40) cigarettes daily. ⿳ㅡㄹ Blood specimens were taken throughout the day from ambulant and non-fasting subjects. Samples were taken into an evacuated tube containing dry $\mathrm{K}_{2}$ EDTA and a full blood count, including an automated differential count, was performed on a Bayer-Technicon H. 2 counter. 
Table 1 Geometric mean and 95\% reference range for total and differential WBC count, platelet count and mean platelet volume in men and women of three ethinic origins

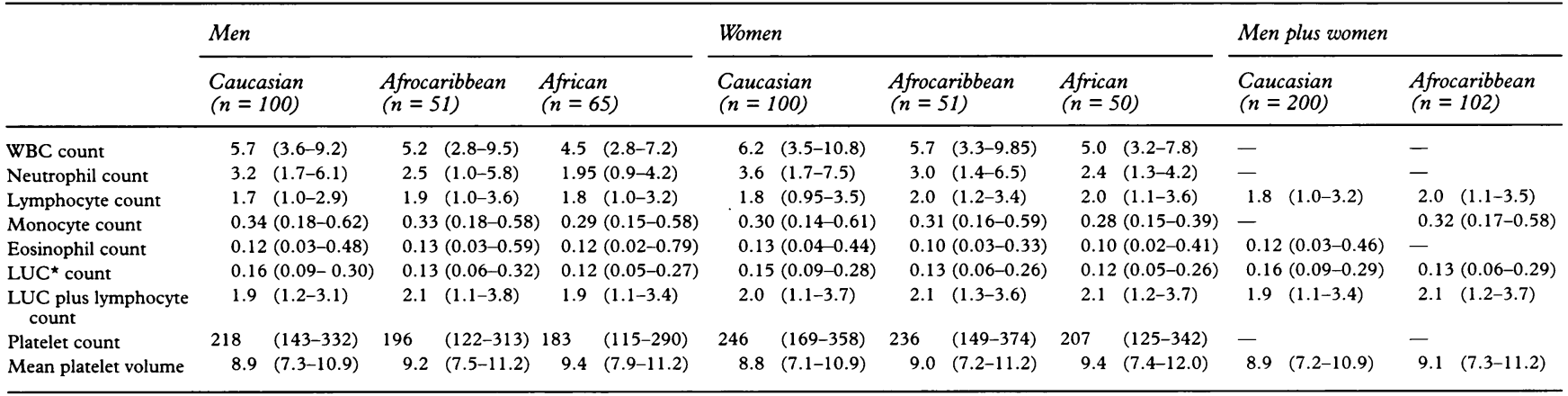

${ }^{\star}$ LUC $=$ large unstained (peroxidase negative) cells.

Table 2 The significance of ethnic and sex differences in total and differential WBC and platelet counts

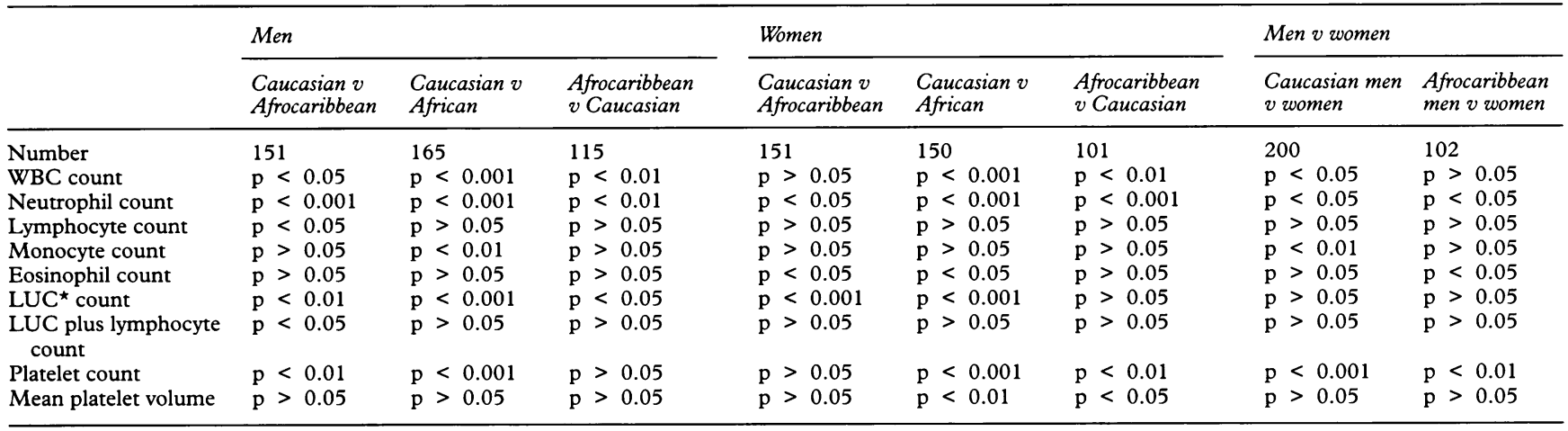

*LUC $=$ large unstained (peroxidase negative) cells.

Data were logarithmically transformed prior to statistical analysis. A geometric mean and $95 \%$ range were derived for the total WBC count, platelet count and absolute counts of neutrophils, lymphocytes, monocytes, and eosinophils. The H.2 counter has a category of large unstained (that is, peroxidase negative) cells (LUCs) which in normal subjects mainly represent large lymphocytes; the counts of lymphocytes plus LUCs were therefore also analysed to give data equivalent to the lymphocyte count with other methodologies. Means for men and women, for smokers and non-smokers and for the three ethnic groups were compared by a Student's $t$ test. When there was no significant difference, results for men and women were pooled.

\section{Results}

Africans and Afrocaribbeans had lower WBC counts and neutrophil counts than Caucasians; Africans had lower counts than Afrocaribbeans (table 1). The differences were statistically significant for all except the Caucasian/Afrocaribbean comparison for WBC count in women (table 2). Africans and Afrocaribbeans showed the same sex differences in the WBC count and neutrophil count that have previously been observed in Caucasians, these differences being statistically significant for all except the WBC count in Afrocaribbeans (tables 1 and 2). The reference ranges for WBC counts and neutrophil counts were sufficiently different from those of Caucasians to make it advisable to use ranges for the appropriate ethnic group when interpreting blood counts. For example, in African men the lowest neutrophil count observed was $0.8 \times 10^{9} / 1$ and the lower reference limit was $0.9 \times 10^{9} / 1$. Similarly, for Afrocaribbean men the lowest neutrophil count observed was $0.8 \times 10^{9} / 1$ and the lower reference limit was $1.0 \times 10^{9} /$. Neither Africans nor Afrocaribbeans had higher eosinophil counts than Caucasians, confirming that the higher counts sometimes observed in Africans are due to parasitic infection or other disease rather than being an ethnic difference. Monocyte counts were similar in the different ethnic groups, although counts tended to be lower in Africans; this difference was significant for the African/Caucasian comparison in men ( $p<0.01)$. Lymphocyte counts were generally similar in the different ethnic groups, although higher counts were observed in African than in Caucasian men $(p<0.05)$.

The lower platelet counts in Africans than in Caucasians, which have been reported previously, were confirmed. Afrocaribbean men were also found to have lower platelet counts than Caucasians (tables 1 and 2). The difference in platelet counts between Caucasian and Afrocaribbean women was not significant. The previously reported sex difference in the platelet count was confirmed for Caucasians and was also demonstrable in Africans and Afrocaribbeans (tables 1 and 2). There was a suggestion that lower platelet counts were associated with a higher mean platelet volume, but this was statistically significant only for the differences between African and Caucasian women and between African and Afrocaribbean women. The difference in the lower limit of the reference range between Africans and Caucasians is sufficient for it to be taken into account when interpreting platelet counts in patients. 
The smokers were found to have higher WBCs counts and neutrophil counts than the non-smokers $(p<0.001$ and $p=0.01$ for WBC counts for men and women, respectively; $p<0.001$ and $p<0.05$ for neutrophil counts for men and women, respectively). Platelet counts did not differ significantly between smokers and non-smokers. The sex differences in WBC, neutrophil and platelet counts were not accounted for by any difference in smoking habits between men and women. Among the 102 non-smokers the WBC, neutrophil and platelet counts were significantly higher in the women than in the men $(p<0.05, p<0.05$, $\mathrm{p}<0.001$, respectively). For the 51 smokers the sex differences were not significant.

\section{Discussion}

The lower WBC counts and neutrophil counts previously reported in Africans and Afrocaribbeans in comparison with Caucasians have been confirmed and reference ranges applicable to subjects of different ethnic origins have been derived. The causes of these differences have yet to be satisfactorily explained. There is some evidence in a Zambian study that counts are higher in those with an urban life style and a more Western diet. ${ }^{1}$ However the Afrocaribbean subjects in the present study had often been born in Britain or had been resident here for many years and it is unlikely that the differences in WBC counts are attributable to dietary or other life style differences. In an earlier study confined to women ${ }^{7}$ differences in the white cell count could not be attributed to differences in oral contraceptive use or cigarette smoking between different ethnic groups. In this study the sex difference in WBC and neutrophil counts was independent of any difference in smoking habits. A genetic explanation is probable. There is limited evidence from this and earlier studies that Africans may have lower monocyte $e^{247}$ and higher lymphocyte counts $^{14}$ than Caucasians but these differences, if real, are not sufficient to be of any practical importance in assessing patient data.

Lower platelet counts, in comparison with Caucasian ranges, have been confirmed for Africans and for Afrocaribbean women. These differences are sufficient to be of practical importance in interpreting counts around the lower end of the reference range. As for the WBC and neutrophil counts, the cause of the lower platelet counts observed in Africans is unknown. The influence of environmental fac- tors or undetected illness is likely to explain part of the difference as Essien $e t a l^{10}$ observed higher counts in "elite" Nigerians than in peasant farmers and as counts reported from $c$ Nigeria $^{10}$ and Zambia $^{9}$ are lower than those@ found for Africans in the present study. However, genetic factors are likely to be relevant.

There is a sex difference in the total WBC, neutrophil and platelet counts which is appar-需 ent in all ethnic groups and seems to be ao genuine biological difference. The cause is흐 unknown. This is of practical importance as $\frac{\bar{s}}{\bar{D}}$ healthy African and Afrocaribbean men have $\triangle$ even lower neutrophil and platelet counts than healthy women of the same ethnic groups.

With the increasing number of blood counts being performed on relatively healthy subjects $\vec{\omega}$ (for example, for genetic counselling or preoperatively) it is important to avoid causing? unnecessary alarm or performing unnecessary $\overrightarrow{0}$ investigations for apparent neutropenia or ${ }^{\circ}$ thrombocytopenia when counts observed do not differ from those usually observed ino healthy subjects of the same sex and ethnic? group. This requires a knowledge of the range of normality in healthy subjects and requireso that haematology laboratories are aware of the $\stackrel{5}{?}$ sex and ethnic origin of a patient when $\vec{\varphi}$ interpreting haematological variables.

The author thanks the 417 volunteers whose kind assistance made this study possible.

1 Ezeilo GC. Genetic neutropenia in Africans. Lancet? 1972;ii: 1003-4.

2 Shaper AG, Lewis P. Genetic neutropenia in people of Afri- 으 can origin. Lancet $1971 ;$ ii: $1021-3$.

3 Rougement A, Boisson M E. Racial differences in the leucocyte count. BMF 1975;2:684-5.

4 Wasserman HP. Leucocyte counts and erythrocyte sedimentation rate in Africa. An interracial study and review of the literature. S Afr Med F 1966;40(Suppl 21):3-24.

5 Cross JP, Heyns ADUP. Haematological reference values for the Basotho. $S$ Afr Med $\mathcal{F} 1983 ; 63: 480-3$.

6 Rippey JJ. Leucopenia in West Indians and Africans. Lancet 1967;ii: 44

7 Bain BJ, Seed M, Godsland I. Normal values for white celli counts in women of four different ethnic origins. $\mathcal{F}$ Clin

Orfanakis NG, Ostlund RE, Bishop CR, Athens JW. Normalo blood leukocyte concentration values. Am $\mathcal{F}$ Clin Pathol 1970;53:647-51.

9 Gill GV, England A, Marshal C. Low platelet count in Zam- O bians. Trans Roy Soc Trop Med Hyg 1979;73:111-12.

10 Essien EM, Usanga EA, Ayeni O. The normal platelet count $N$ and platelet factor 3 availability in some Nigerian popula-tion groups. Scand $\mathcal{f}$ Haematol 1973;10:378-83.

11 Mukibi JM, Okelo GBA, Kanja C. Platelet counts in normal Kenyan adults. East Afr Med $\mathcal{f} 1981 ; 58$ : 136-9. 2 Bain BJ, Seed M. Platelet count and platelet size in Africans and West Indians. Clin Lab Haematol 1986;8:43-8.

13 Bain BJ, England JM. Normal haematological values: sex difference in the neutrophil count. BMF 1975;11:473-5. के

14 Bain BJ. Platelet count and platelet size in men and women. Scand $\mathcal{F}$ Haematol 1985;35:77-9. 